\title{
Reach and representativeness of ethnic minority women in the Health Is Power Study: a longitudinal analysis
}

\author{
Rebecca E. Lee, PhD, ${ }^{1}$ Jacqueline Y. Reese-Smith, PhD, ${ }^{2}$ Scherezade K. Mama, DrPH, ${ }^{3}$ Ashley V. Medina, BS, ${ }^{4}$ \\ Kristin L. Wolfe, BA, ${ }^{4}$ Paul A. Estabrooks, $\mathrm{PhD}^{5}$
}

${ }^{1}$ Center for Health Promotion and Disease Prevention, College of Nursing and Health Innovation, Arizona State University, 550 N 3rd St, Phoenix, AZ 85004, USA

${ }^{2}$ Department of Psychology, Jackson State University, 1400 J.R. Lynch Street, LIB Rm 232, Jackson, MS 39217, USA

${ }^{3}$ Department of Kinesiology, The Pennsylvania State University, 268) Recreation Building, University Park, PA 16802, USA

${ }^{4}$ Texas Obesity Research Center, Department of Health and Human

Performance,

University of Houston, 3855 Holman Street, Garrison Gym Rm 104, Houston, TX 77204-6015, USA

${ }^{5}$ Department of Health Promotion, Social \& Behavioral Health, College of Public Health,

University of Nebraska Medical

Center, 984365 Nebraska Medical Center, Omaha, NE 68198, USA

Correspondence to: $\mathrm{R}$ Lee

releephd@yahoo.com

Cite this as: TBM 2017;7:106-114 doi: 10.1007/s13142-016-0385-9

\section{Abstract}

Reach is a key factor in translating research to practical application. This study examined reach and representativeness of a multi-city, randomized controlled community health trial in African American (AA) and Hispanic or Latina $(\mathrm{HL})$ women. Participants completed measures of demographics, body mass index (BMI), percent body fat, resting heart rate, and blood pressure followed by a run-in procedure and a randomization meeting. AA were more likely to be screened out initially; $\mathrm{HL}$ were more likely to drop out. Participation did not differ by city or recruitment method. Women who completed the post-intervention assessment were more likely to be AA, older, and have higher socioeconomic status ( $p$ values <.05). This study showed moderate levels of reach but overrepresented higher educated, wealthier, and older women at the completion of the study. Representativeness can change over the course of the study and impact the practicality of translating research to practice.

\section{Keywords}

Reach, Representativeness, African American, Hispanic, Minority health, Women

Improving the reach and representativeness of ethnic minority communities in health promotion studies has been a focus of US funding agencies and researchers alike for over two decades, as it represents a key factor in the viability of translating research findings to broad-based implementation in vulnerable populations. Despite this greater emphasis on representing people from ethnic minority groups in research, relatively few reports exist that measure and report these factors in detail in clinical and behavioral research studies [1-9]. Reach represents not only the absolute number of participants-the " $N$ "-but also the proportion of those recruited to those eligible to participate and the representativeness of the sample, not only at recruitment, but throughout the study [10]. Reach is a broadly defined construct that results from effective recruitment and retention [11].

A growing body of literature has addressed strategies to enhance recruitment, such as overcoming barriers like lack of interest, lack of knowledge of the topic under investigation, higher rates of exclusion

\section{Implications}

Better reporting on challenges, resources, and effective strategies used to recruit and retain representative samples is needed to inform research and practice among minority populations.

for medical reasons, and a wide array of individual beliefs and psychosocial factors [12-17]. Most studies report the number of people recruited, perhaps reporting recruitment ratios (number recruited/ number eligible or screened) [5, 18-20]. In contrast, very few studies report characteristics indicating the representativeness of the sample and whether the sample that was retained through completion of the study represented the sample that was recruited initially [21]. Representativeness is a particularly important consideration as the findings can only be generalized to those represented in the study, affecting the practicality of translating research to action. People from lower socioeconomic status backgrounds and ethnic minorities may be more likely to dropout compared to wealthier and white populations, often attributed to dissatisfaction with the research experience such as lack of motivation, dissatisfaction with results, feelings of abandonment, a lack of encouragement or poor social support, the belief one can accomplish the study goal without assistance, other personal reasons (e.g., work interference, lack of time, stress), healthcare system issues (e.g., lack of flexibility in scheduling appointments), and study design (e.g., difficult study regimes) [1, 22-28].

Recruiting and retaining a representative sample are both equally important challenges for researchers [4]. In fact, attrition is the beginning of a spiral effect in which valuable information is lost, researchers are unable to recoup limited research dollars spent on the initial participation, and reduced power owing to smaller sample sizes creates threats to valid data analysis, compromising internal validity and contributing to spurious findings $[13,29]$. This contributes to poor ecologic validity and inability to generalize results [24, 29, 30]. As many as a third of published studies do not report criteria used to determine attrition, or only provide minimal descriptions of sample attrition, making it difficult to compare representativeness 
across studies and samples. Reduced representativeness of the target population affects the likelihood of results being useful for efforts at scaling up the research methodology for broad-based implementation. Recommendations suggest that studies should report information systematically documented by ethnicity, to compare how the study sample represents the population at recruitment and throughout the study, along with SES, gender, and age [7, 21, 31]. The goal of this study was to evaluate reach and representativeness in the Health Is Power (HIP) study by (1) evaluating recruitment and participation rates, (2) determining the degree to which the initial study sample represented the population demographically, and (3) determining the degree to which those who completed the intervention represented the original study sample.

\section{METHOD}

\section{Participants}

The HIP study recruited African American and Hispanic or Latina women between the ages of 25 and 60 years old who were residents in Harris or Travis County, Texas to participate in a physical activity or dietary habits randomized controlled trial (CA109403) [3237]. Women who were physically active or unable to pass the Physical Activity Readiness Questionnaire (PAR-Q) or did not meet all inclusionary criteria were ineligible to participate in the study [37]. All study protocols and procedures were approved by the Committee for the Protection of Human Subjects at the University of Houston and the Institutional Review Board at the University of Texas, Austin, and participants provided written informed consent to participate.

\section{Procedures}

Participant recruitment-Participants were recruited using a number of methods to draw a diverse sample of women. Face-to face announcements of the HIP study were made by a member of the research team or a community liaison at numerous community events and meetings, and advertisements or announcements were posted with local newspapers, magazines, television stations, and websites that targeted African American and Hispanic or Latina women to enroll participants in the HIP study. The advertisements shared basic information about the study, listed brief inclusion criteria, and provided the principal investigator's contact information. Interested participants were instructed to call for more information about enrollment in the HIP study and to complete a telephone screening.

Eligibility and screening procedures-At the start of the screener, participants gave a brief verbal consent to complete the screener and endorsed that participation in the HIP study was voluntary. Trained research team members used a script outlining the details of the project, and administered the PAR-Q to determine eligibility.[38] Women were then screened to meet the following inclusion criteria: (1) self-identified as
African American or Hispanic or Latina, (2) between the ages of 25 and 60 years old (to include adults outside college and retirement age ranges), (3) able to read, speak, and write in English or Spanish, (4) not pregnant or planning to become pregnant within the next 12 months, (5) a Harris or Travis County resident, (6) no plan to move within the next 12 months, (7) physically inactive or reportedly less than $30 \mathrm{~min}$ of physical activity per day on 3 or more days per week, (8) free from medical illness nor currently taking medication contraindicated for participation in moderate intensity physical activity, (9) willing to be randomized to either a physical activity or a vegetable and fruit intervention group, and (10) available between 5:30 and 8:00 PM on meeting dates. Women who did not meet criterion number 8 were allowed to enroll in the study if they obtained a medical waiver from their physician stating they were able to participate in HIP under the physician's supervision. Those who did not meet one or more of the screening criteria, excluding criterion 8 , were excluded from the HIP study.

Women who met inclusion criteria were invited to complete the time 1 (T1) baseline health assessment. Ineligible women were invited to leave their contact information for a prospective participant pool database for future research opportunities with the research group. T1 health assessment appointments were made on a specified date, and individual appointments were scheduled at the participants' convenience on that date. Upon arrival to the T1 assessment, participants reviewed study details and signed an informed consent.

Assessments, run-in, and intervention-At the T1 assessment, participants completed an intervieweradministered questionnaire, measured resting heart rate and blood pressure, anthropometric measures of body mass index $\left(\mathrm{BMI}=\mathrm{kg} / \mathrm{m}^{2}\right)$ and body fat, and then were given a take-home packet of selfadministered questionnaires to complete during a 2week run-in phase. Given higher than expected dropout rates among women of color in previous studies, multiple strategies were used to encourage less committed participants to drop out prior to randomization [37]. The first of these strategies was the 2 -week run-in phase, designed to minimize attrition in the remainder of the study prior to randomization, enhance commitment to the project, and demonstrate willingness to be randomized. The run-in phase included the completion of a packet of questionnaires, health behavior monitoring logs, and attendance at a randomization session.

The second strategy occurred at the randomization session, where women were encouraged to consider the importance of the HIP study and the role that random assignment plays in internal validity and reducing bias for the study [39]. Women used small group activities to explore the reality that both groups (physical activity and fruit and vegetable) had advantages and disadvantages. Women were encouraged to consider possible reasons why dropout occurs, to 
1948 Women were phone screened for eligibility

1003 Were not eligible

328 Unable to schedule and/or attend baseline assessment

317 Have a health compromising condition that may be aggravated by physical activity

163 Currently physically active

82 Not a Harris or Travis County resident

52 Schedule conflict and/or could not attend intervention sessions

37 Planning to move within the next 12 months

12 Pregnant or planning to become pregnant in the next 12 months

6 Not African American or Hispanic or Latina woman between the ages of 25 and 60 years old

4 Unwilling to be randomized to either group

2 Unable to read, write and/or speak English or Spanish

$\downarrow$

945 Eligible and scheduled to attend baseline time 1 (T1) health assessment

692 African American women

253 Hispanic or Latina women

154 Austin

99 Houston

410 Completed the $\mathrm{T} 1$ health assessment

258 African American

152 Hispanic or Latina

103 Austin

49 Houston

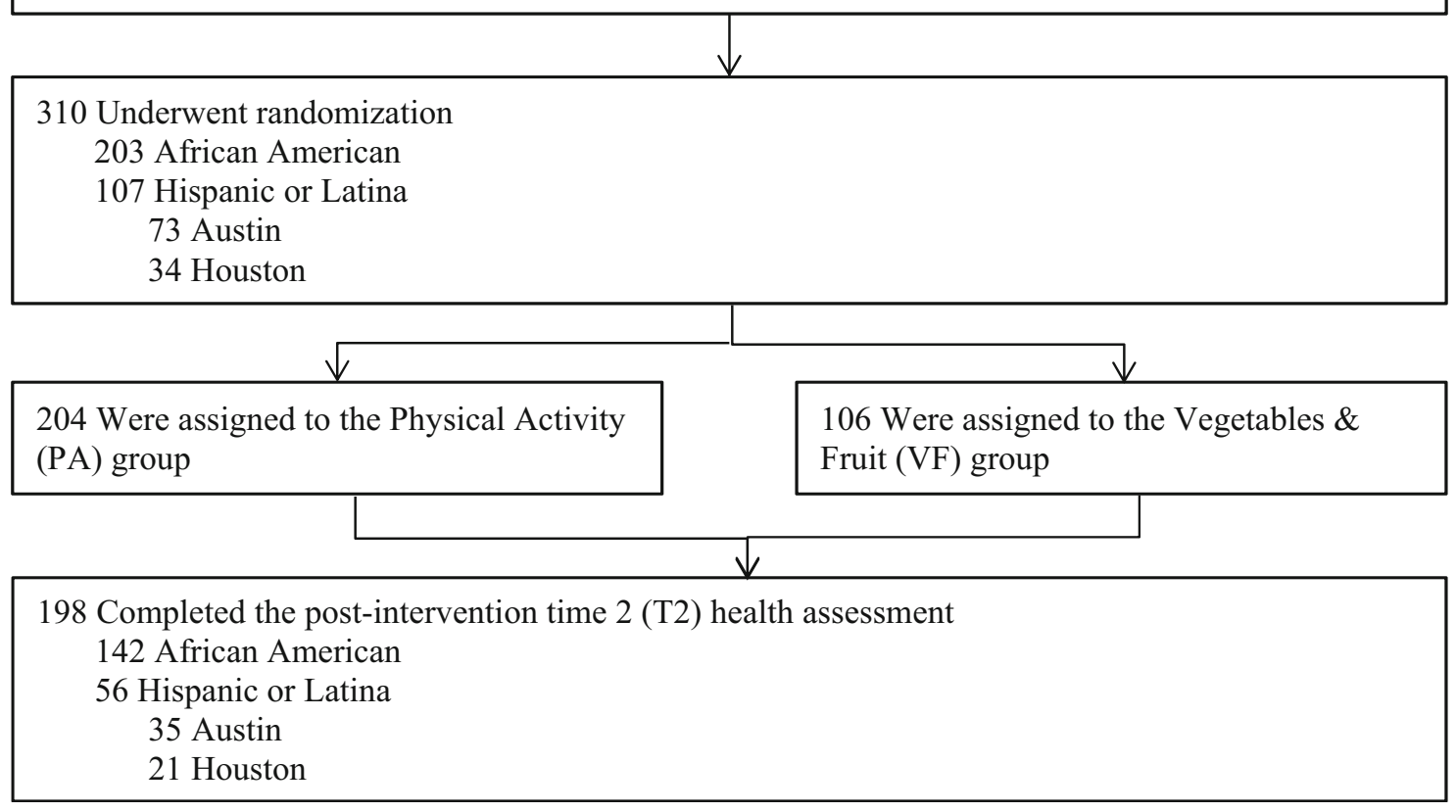

Fig. 1 | Flow of participants through the Health Is Power study 
consider their calendars for the next 12 months and to avoid enrolling in the HIP study at that time if they knew that they had conflicts with future meetings. Women also were asked to withdraw before randomization if they were not completely comfortable with being assigned to either group. After this exercise, women were randomized to a physical activity or vegetable and fruit group using a double-blind randomization procedure.

Women met with their assigned groups six times over the course of 6 months, and groups were exposed to a health promotion intervention to promote walking or increase vegetable and fruit consumption that has been previously described [33, 37]. The first three sessions were 2 weeks apart; sessions 4 through 6 were scheduled approximately 1 month apart. Attendance was taken at each session to monitor retention and attrition throughout the study. After 6 months of intervention sessions, participants completed a time 2 (T2) post-intervention health assessment.

\section{Measures}

Demographics-Participants reported their age and ethnicity. Information assessing household income, educational attainment, and parental educational attainment was collected from the Maternal and Infant Health Assessment (MIHA) survey [40]. Items from this survey have been used successfully with samples representing a diverse range of ethnicities and socioeconomic status categories [41].

Similar demographic measures were used from the 2000 United States Census, ethnic/racial minority group (white, black/African American, American Indian/Alaska Native, Asian, Other, Mixed, Hispanic/Latino), including median household income and percent below or above the Federal Poverty Level, and educational attainment. All data were aggregated at the census tract level for each participant's home address [42].

Physical assessments-Physical health assessments (T1, T2) included measures of resting heart rate, blood pressure, height, weight, and percent body fat. Resting heart rate and blood pressure were collected by trained personnel using established protocols [43]. Height was measured using a portable stadiometer. Body weight, BMI, and percent body fat were measured using bioelectrical impedance analysis using a Tanita TBF-310 body composition analyzer [44]. Measurements were assessed twice to ensure accuracy, and an average of the two measurements was used in analyses.

\section{Statistical analyses}

Descriptive analyses were used to characterize participants in terms of sociodemographic factors at all study time points. Frequencies were used to report participation and attrition rates throughout the study in order to evaluate recruitment and retention rates. Chi-square analyses were used to determine whether recruitment or participation varied by recruitment strategy.

In order to determine whether the study sample was representative of the population, we compared descriptive characteristics of our sample with 2000 U.S. Census data at the tract level. We extracted data for ethnicity, educational attainment, and median income and percent below and above the federal poverty level for the total population in Harris and Travis Counties, as these variables were conceptually similar to those that were obtained during the $\mathrm{T} 1$ health assessment.

Chi-square $\left(\chi^{2}\right)$ and independent sample $t$ test analyses by demographic and physical health indicators believed to influence participation were used to determine whether the sample that completed the T1 health assessment, run-in procedure, and attended the randomization session differed from those who completed the assessment and run-in procedures but did not attend randomization. To determine whether the post-intervention study sample was representative of the entire sample initially recruited at $\mathrm{T} 1$ in terms of demographics and physical health indicators, we again conducted independent samples $t$ tests comparing T2 completers and non-completers. All analyses were completed using IBM SPSS 20.0 and significance was set at $\alpha=0.05$ level [45].

\section{RESULTS}

\section{Recruitment}

The most commonly reported source for how women had heard about the study was from a newspaper or magazine article $(45.3 \%, n=404)$, followed by a referral from a friend or relative $(33.8 \%, n=301)$. Less commonly reported sources were from an email, listserv or on the Internet $(30.5 \%, n=97)$, places visited such as university campus, church or a health fair $(14.2 \%, n=38)$, and television or radio $(7.4 \%, n=$ 66). More African American women reportedly heard about the study on campus, at church, or at a health fair (57.9 vs. $42.1 \%, X^{2}=4.332, d f=1, p=.037$ ) than Hispanic or Latina women. The retention of participants was not associated with recruitment strategy $(p>.05)$.

\section{Reach}

As illustrated in Fig. 1, 945 women were phone screened eligible to participate and invited to the T1 health assessment. Of these, 410 attended the T1 health assessment, 310 were randomized, and 198 were retained at T2. Only $43 \%$ of those screened eligible showed for their scheduled $\mathrm{T} 1$ health assessment, and only $33 \%$ of those screened were randomized, suggesting a screening to randomization ratio of 3 to 1 . Of those who were screened eligible, $21 \%$ were retained at $\mathrm{T} 2$, and of those who were randomized, $64 \%$ were retained at $\mathrm{T} 2$.

Among the 692 African American women who were screened eligible, 258 attended the T1 health assessment, 203 were randomized, and 142 were 
retained at T2. About $37 \%$ of those screened showed for their scheduled $\mathrm{T} 1$ health assessment, and only $29 \%$ of those screened were randomized, suggesting a lower screening to randomization ratio among African American women. Of those who were screened, $20 \%$ were retained at $\mathrm{T} 2$, and of those who were randomized, $70 \%$ were retained at $\mathrm{T} 2$.

Of the 253 Hispanic or Latina women who were screened eligible, 152 attended the T1 health assessment, 107 were randomized, and 56 were retained at T2. About $59 \%$ of those screened showed for their T1 health assessment, and $39 \%$ of those screened were randomized. Of those who were screened, $22 \%$ were retained at $\mathrm{T} 2$, and of those who were randomized, $52 \%$ were retained at $\mathrm{T} 2$.

Significantly fewer eligible African American women showed for the T1 health assessment compared to eligible Hispanic or Latina women $\left(\chi^{2}(1)=5.042\right.$, $p=.025)$. Although there was no difference by ethnicity at randomization, significantly more African American women returned for T2 compared to Hispanic or Latino women $\left(\chi^{2}(1)=8.663, p=.003\right)$. Note that fewer Hispanic or Latina women were recruited in this study, because unexpected budget shortfalls necessitated stopping the Hispanic or Latino arm of the study early.

\section{Sample representativeness at $\mathrm{T} 1$}

Sample characteristics for women who completed T1 are presented by ethnicity in Table 1. African American and Hispanic or Latina women were similar in age, income, BMI, and percent body fat. However, African American women had higher educational attainment $\left(X^{2}=61.415, d f=2, p<.001\right)$ and higher systolic $(t=2.447, p=.015)$ and diastolic $(t=2.372$, $p=.018)$ blood pressure and lower resting heart rate $(t=-2.252, p=.025)$ than Hispanic or Latina women at $\mathrm{T} 1$.

The focus of the study on African American and Hispanic or Latina women was appropriate for the regional population. Harris County is $18 \%$ nonHispanic black or African American and $38 \%$ Hispanic or Latino. Travis County is $8 \%$ black or African American and $32 \%$ Hispanic or Latino. The sample differed from the regional population on economic dimensions, with only $4 \%$ of participants living below the poverty level compared to $17 \%$ of the regional population. The study sample was also not representative on measures of education, with $54 \%$ of the African American sample and $29 \%$ of the Hispanic or Latina sample having completed college compared to $3 \%$ of African Americans and $1.0 \%$ of Hispanics in the region.

Representativeness of retained participants after the run-in procedure

Women who completed T1 and returned for the randomization session tended to be slightly older $(M=$ $45.8, \mathrm{SD}=9.1)$ than those who did not return $(M=$ $43.5, \mathrm{DS}=9.8, t(395)=-2.131, p=.034)$. There were no other differences in demographics or physical health indicators believed to influence participation at $\mathrm{T} 1$ between those who completed the run-in procedure and those who did not.

\section{Representativeness of retained participants at T2}

As presented in Fig. 2, women who were retained at T2 had higher educational attainment $(54.2 \%$ of completers were college graduates vs. $35.3 \%$ of noncompleters, $\left.\chi^{2}=15.475, d f=2, p<.001\right)$ and higher income $(56 \%$ of completers reported an income $401+\%$ above the federal poverty level vs. $40.9 \%$ of non-completers, $\chi^{2}=13.371, d f=4, p=.010$ ) at $\mathrm{T} 1$, compared to those who did not complete the study or attend the T2 assessment. On health measures, women who were retained were older $(m=47.0, \mathrm{SD}=$ 8.5 vs. $m=43.5, \mathrm{SD}=9.7$ years, $t=-3.785, p<.001)$ and had lower resting heart rate $(m=72.4, \mathrm{SD}=8.3 \mathrm{vs}$. $m=74.7, \mathrm{SD}=9.2$ beats per minute, $t=2.551$, $p=.011)$ at T1 compared to non-completers. Women who completed $\mathrm{T} 2$ did not differ at $\mathrm{T} 1$ on any other demographic or physical health indicators.

\section{DISCUSSION}

Reaching sizable, diverse, and representative samples of ethnic minority women for health promotion studies remains a challenging and resource-intensive

Table 1 | Time 1 sample characteristics by ethnicity in the Health Is Power study

\begin{tabular}{llll} 
& \multicolumn{1}{c}{ African American } & Hispanic or Latina & Total \\
& $N=258$ & $N=152$ & $N=410$ \\
& $M(\mathrm{SD})$ & $M(\mathrm{SD})$ & $M(\mathrm{SD})$ \\
\hline Age (years) & $44.9(9.4)$ & $46.1(9.2)$ & $45.3(9.3)$ \\
\hline Systolic blood pressure* & $126.4(13.9)$ & $122.9(13.3)$ & $125.2(13.8)$ \\
\hline Diastolic blood pressure* & $79.6(9.6)$ & $77.2(9.7)$ & $78.8(9.7)$ \\
\hline Resting heart rate* & $72.8(8.6)$ & $74.8(9.1)$ & $73.5(8.8)$ \\
\hline BMl $\left(\mathrm{kg} / \mathrm{m}^{2}\right)$ & $35.1(9.3)$ & $34.1(6.9)$ & $34.7(8.6)$ \\
\hline Percent body fat & $43.1(7.5)$ & $42.4(6.4)$ & $42.8(7.1)$ \\
\hline & $\%(\mathrm{~N})$ & $\%(\mathrm{~N})$ & $\%(\mathrm{~N})$ \\
\hline Completed college* & $53.7(132)$ & $28.6(40)$ & $44.6(410)$ \\
\hline Income $401 \%$ above the federal poverty line* & $53.4(124)$ & $40.6(54)$ & $48.8(410)$ \\
\hline${ }^{*} p$ <.05 & &
\end{tabular}




\section{a Educational Attainment}

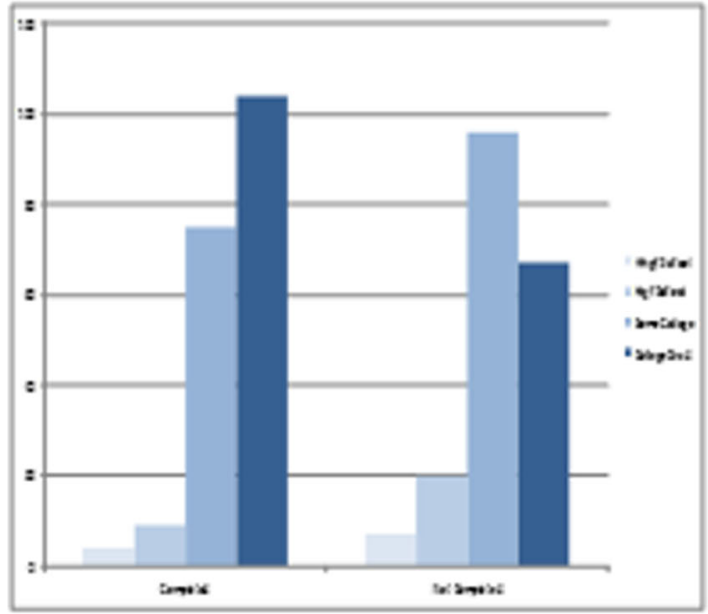

c

Age

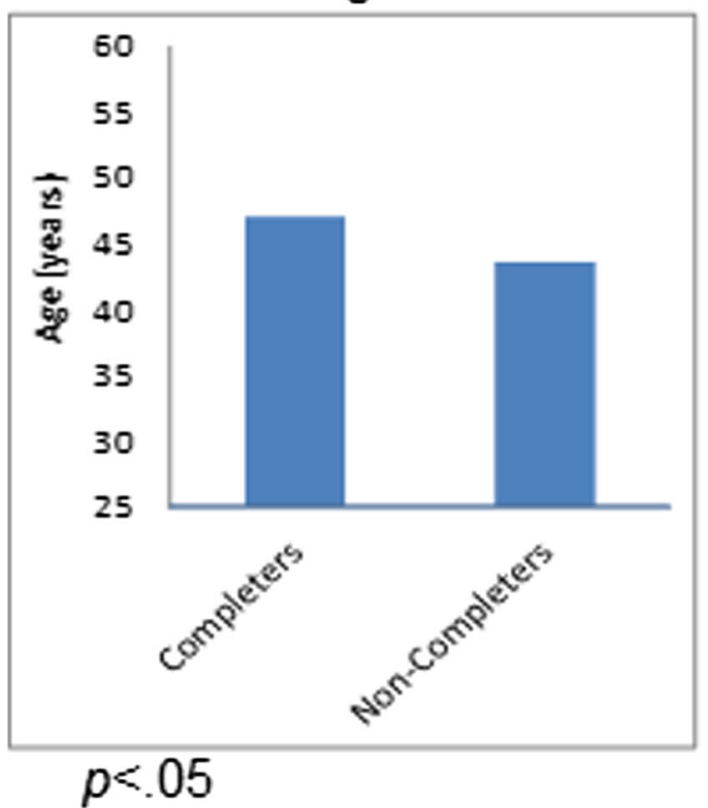

b Standardized Income (\% of FPL)

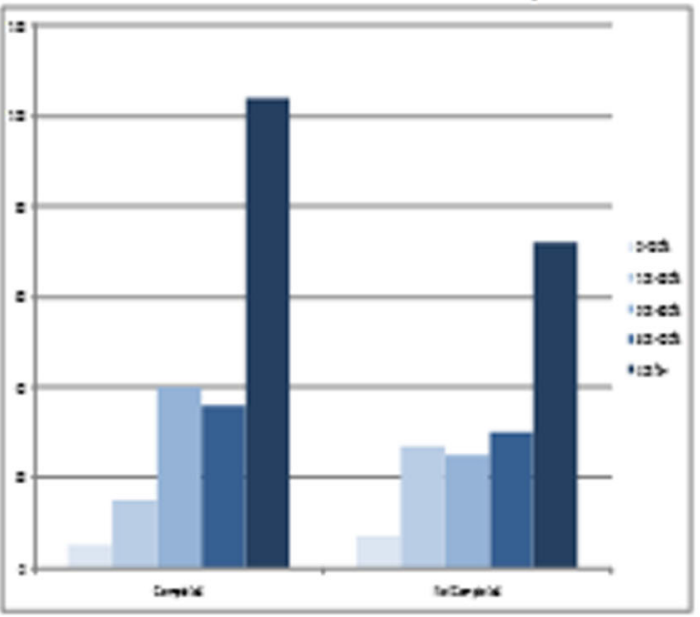

d Resting Heart Rate

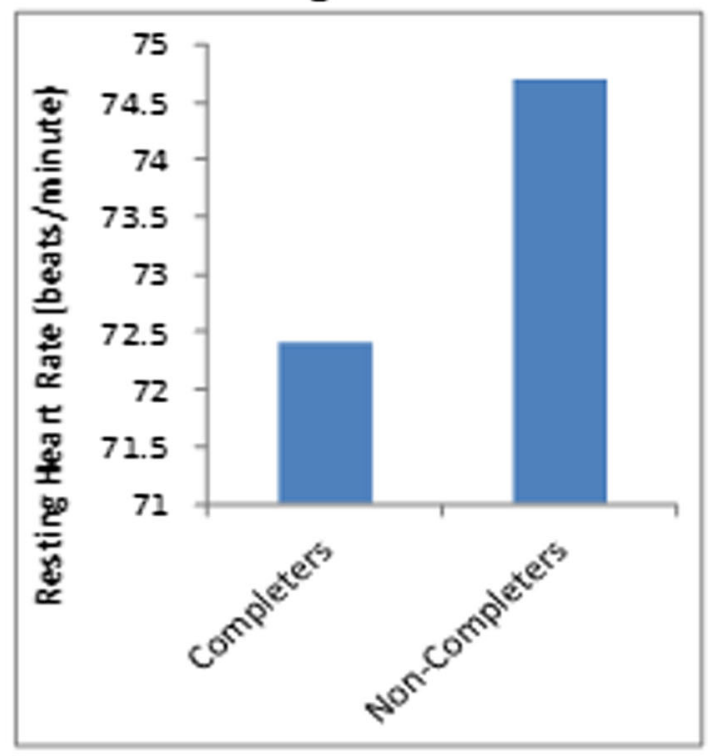

Fig. 2 | Differences between women who completed the intervention compared to those who did not

proposition and poses a challenge for efforts to translate meaningful trial research for broad-based scalability. Among African American and Hispanic or Latina women in this sample, only about one in five of the women who were originally recruited were retained at the primary outcome measurement endpoint, which is consistent with other randomized controlled trials in the behavioral and clinical sciences [12, 14, 19, 46, 47]. Also, similar to other studies, most participants dropped out early in the process, owing likely to both the complexity of behavior change and the efficacy of strategies employed herein to encourage drop out early in the study [48-51]. This also suggests that the representativeness of initially recruited samples may differ from the representativeness of the eventual retained sample. In this study, nearly one fourth of participants dropped out between $\mathrm{T} 1$ and randomization. Since previous studies have reported high attrition rates for minority women early in randomized controlled trials, it makes sense to employ strategies to encourage this for efficacy trials, prior to investing many resources in the sample under investigation [49, 52, 53]. It may make sense to recruit an oversample of people who are more likely to attrite, to maintain representativeness after the intervention is complete.

African American women tended to have a faster rate of dropout early on in the project, during the period from recruitment to randomization, compared to Hispanic or Latino women. Regardless of these early differences between the samples, both groups participated similarly in early project activities. In contrast, more African American women reached the final measurement endpoint. The better screening to randomization ratio for Hispanic or Latino women may suggest higher initial compliance, but their higher 
attrition ratio in contrast may mean that stronger or different retention methods or incentives are needed for this population.

Multiple modes of contact, screenings, and run-in strategies appear to be appropriate and useful to reach minority women in efficacy studies. However, these strategies bear deeper consideration for effectiveness studies. If, despite numerous strategies to cast as broad a net as possible, it is still most challenging to reach the most vulnerable of populations for individually focused interventions, perhaps, intervention and treatment strategies are needed to reach people without having to participate in intensive screenings to meet the enrollment criteria for traditional interventions. A second consideration in population reach is the overrepresentation of highly educated women in this sample, compared to the regional population. This may in part be attributed to the investigators being based in a university setting, and, as such, all press and marketing about the study was generated and had to be approved by University sources. Efforts were made to reach a more representative sample by partnering with community members and organizations. Although these kind of participatory action research strategies aim to increase connection to the community by increasing understanding of the value of the research and enhancing communication and dissemination to a broader audience, we were not successful at reaching a broader audience [49, 50, 54].

Women who completed the intervention were different on several important domains compared to those who completed their initial assessments, demonstrating how representativeness can change over the course of the study. That is, the initially recruited sample may represent a different population than the sample who actually completed the study. Women who completed tended to have higher educational attainment, higher incomes, and were a bit older. Significant existing personal resources may be important for completing typical health intervention trials; thus, this may be another contributing factor to understanding the underrepresentation of some groups of the population. Some researchers have suggested that many research participants may not fully realize what is involved in participating in a randomized controlled trial, despite informed consent and orientation procedures [48]. Consider that in this trial, similar to many of its kind, in order to participate in this study, women had to attend a total of two health assessments, one randomization session and six intervention sessions, for a total of nine sessions in 6 months. Sessions were held in the evenings, and no child care was provided, because meeting university liability concerns was not feasible within the constraints of the budgeted resources. Some women did bring children with them, and health educators made the children comfortable and distributed coloring books and crayons that had been donated by a community partner. The study also offered metro passes to women, but only one participant accepted this offer, suggesting that most women preferred to drive or use other transportation. The study also offered free parking. Regardless of these measures, women would need to have sufficient time to attend, resources for transportation (money for gasoline and automobile costs), as it seemed that most women drove their own cars, and resources for managing other family members and personal responsibilities in the evening, if they chose not to bring them with them, while they were away. These considerations would need to be included on top of intervention requirements that included increasing daily physical activity or fruit and vegetable consumption. Intervention content addressed many commonly reported barriers, such as lack of time, competing demands, lack of social support, family commitments, and safety, and intervention time was devoted to small group works on overcoming barriers with significant time devoted to problem solving; however, these barriers may still have impeded women's ability to complete intervention activities and contributed to attrition. The sample that was retained was higher SES drawn from an already high SES sample. There may have been SES-related barriers, but the traditional barriers of lower SES women might not have applied to this sample. A follow-back study of these women suggested that concerns about caregiving were very important, and that physical activity was simply a lower priority [37]. Successful retention has been accomplished with greater staff time, support and attention, social support and time with peers during the intervention, interventions with less time commitment, increased remuneration, commitment to finish, and understanding of the importance of the study as reasons for completion, suggesting possible additional avenues for improved retention strategies [12, 17, 23, 55-58]. Although many of these considerations were included in this study, it is clear that there is still room for future studies to investigate whether participant burden can be minimized sufficiently to include harder to reach and underrepresented populations or whether different kinds of strategies are needed that simply remove individual, participant burden by focusing on policy, environment, and systems.

This study found reasonable reach and retention rates but poor representativeness of African American and Hispanic or Latino women. Strengths included a sizable sample of minority women in a diverse geographic region, strong measures, and clear documentation of how the representativeness of the sample changed throughout the study. Effective implementation of a run-in and randomization procedure helped those likely to drop out to do so early in the process, reducing dropouts after randomization to intervention groups. Run-in strategies work well for behavioral efficacy interventions but may sacrifice representativeness. The sample was more educated and had higher income compared to regional averages. Women who completed the study had higher educational attainment and income and were older compared to those who did not complete the study, suggesting research requires significant resources at multiple levels-participant, scientist, and community-in order to improve reach and representativeness. 
Acknowledgments: This research was funded by a grant awarded to Dr. Rebecca E. Lee from the National Cancer Institute of the National Institutes of Health (No. 1R01CA109403).

1. BeLue R, Taylor-Richardson KD, Lin J, Rivera AT, Grandison D. African Americans and participation in clinical trials: differences in beliefs and attitudes by gender. Contemp Clin Trials. 2006; 27(6): 498-505.

2. Caban CE. Hispanic research: implications of the National Institutes of Health guidelines on inclusion of women and minorities in clinical research. J Natl Cancer Inst Monogr. 1995; 18: 165-169.

3. Chen MS, Wolle J, Affonso DD, et al. Recruitment of minority communities of task group IV. In Health Behavior Research in Minority Populations: Access, Design, and Implementation (NIH Pub No. 922965). Bethesda, MD: National Institutes of Health; 1992.

4. Keller CS, Gonzales A, Fleuriet KJ. Retention of minority participants in clinical research studies. West I Nurs Res. 2005; 27(3): 292-306.

5. Lee RE, McGinnis KA, Sallis JF, Castro CM, Chen AH, Hickmann SA. Active vs. passive methods of recruiting ethnic minority women to a health promotion program. Ann Behav Med. 1997; 19(4): 378-384.

6. Levkoff SE, Levy BR, Weitzman PF. The matching model of recruitment. J Men Health Aging. 2000; 6: 29-38.

7. Napoles AM, Chadiha LA. Advancing the science of recruitment and retention of ethnically diverse populations. Gerontologist. 2011; 51(Suppl 1): S142-S146.

8. National Institutes of Health: NIH Guidelines on the inclusion of women and minorities as subjects in clinical research. NIH Guide. 1994; 23(11).

9. UyBico SJ, Pavel S, Gross CP. Recruiting vulnerable populations into research: a systematic review of recruitment interventions. I Gen Intern Med. 2007; 22(6): 852-863.

10. Applying the RE-AIM Framework. Available at http://cancercontrol. cancer.gov/IS/reaim/applyreaim.html.

11. Estabrooks PA, Allen KC. Updating, employing, and adapting: a commentary on what does it mean to "employ" the RE-AIM model. Eval Health Prof. 2013; 36(1): 67-72.

12. Eakin EG, Bull SS, Riley K, Reeves MM, Gutierrez S, McLaughlin P. Recruitment and retention of Latinos in a primary care-based physical activity and diet trial: the resources for health study. Health Educ Res. 2007; 22(3): 361-371.

13. Frierson GM, Williams DM, Dunsiger S, et al. Recruitment of a racially and ethnically diverse sample into a physical activity efficacy trial. Clin Trials. 2008; 5(5): 504-516.

14. Kumanyika SK, Whitt-Glover MC, Gary TL, et al. Expanding the obesity research paradigm to reach African American communities. Prev Chronic Dis. 2007; 4(4): A112.

15. Napoles-Springer AM, Fongwa MN, Stewart AL, Gildengorin G, PerezStable E). The effectiveness of an advance notice letter on the recruitment of African Americans and Whites for a mailed patient satisfaction survey. I Aging Health. 2004; 16(5 Suppl): 124S-136S.

16. Nitkin R. Patient recruitment strategies training workshop conducted by the National Institutes of Health. MD: Bethesda; 2003.

17. Yancey AK, Ortega AN, Kumanyika SK. Effective recruitment and retention of minority research participants. Annu Rev Public Health. 2006; 27: 1-28.

18. Kannan S, Sparks AV, Webster JD, Krishnakumar A, Lumeng J. Healthy eating and harambee: curriculum development for a culturally-centered bio-medically oriented nutrition education program to reach African American women of childbearing age. Matern Child Health I. 2010; 14(4): 535-547.

19. Rieder J, Khan UI, Heo M, et al. Evaluation of a community-based weight management program for predominantly severely obese, difficult-to-reach, inner-city minority adolescents. Child Obes. 2013; 9(4): 292-304

20. Two Feathers J, Kieffer EC, Palmisano G, et al. Racial and Ethnic Approaches to Community Health (REACH) Detroit partnership: improving diabetes-related outcomes among African American and Latino adults. Am J Public Health. 2005; 95(9): 1552-1560.

21. Galaviz KI, Harden SM, Smith E, et al. Physical activity promotion in Latin American populations: a systematic review on issues of internal and external validity. Int / Behav Nutr Phys Act. 2014; 11: 77.

22. Brannon EE, Kuhl ES, Boles RE, et al. Strategies for recruitment and retention of families from low-income, ethnic minority backgrounds in a longitudinal study of caregiver feeding and child weight. Child Health Care. 2013; 42(3): 198-213.

23. Grossi E, Dalle Grave R, Mannucci E, et al. Complexity of attrition in the treatment of obesity: clues from a structured telephone interview. Int J Obes. 2006; 30(7): 1132-1137.

24. Janson SL, Alioto ME, Boushey HA. Attrition and retention of ethnically diverse subjects in a multicenter randomized controlled research trial. Control Clin Trials. 2001; 22(6 Suppl): 236S-243S.

25. Senturia YD, McNiff Mortimer K, Baker D, et al. Successful techniques for retention of study participants in an inner-city population. Control Clin Trials. 1998; 19(6): 544-554.
26. Teixeira PJ, Going SB, Houtkooper LB, et al. Pretreatment predictors of attrition and successful weight management in women. Int J Obes Relat Metab Disord. 2004; 28(9): 1124-1133.

27. van der Waerden JE, Hoefnagels C, Jansen MW, Hosman CM. Exploring recruitment, willingness to participate, and retention of low-SES women in stress and depression prevention. BMC Public Health. 2010; 10: 588.

28. Wilbur J, Vassalo A, Chandler P, McDevitt J, Miller AM. Midlife women's adherence to home-based walking during maintenance. Nurs Res. 2005; 54(1): 33-40.

29. Shadish WR, Cook TD, Campbell DT. Experimental and quasiexperimental designs for generalized causal inference. Belmont: Wadsworth; 2002.

30. Duyk G. Attrition and translation. Science. 2003; 302 (5645): 603-605.

31. Davis MJ, Addis ME. Predictors of attrition from behavioral medicine treatments. Ann Behav Med. 1999; 21(4): 339-349.

32. Layne CS, Mama SK, Banda JA, Lee RE. Development of an ecologically valid approach to assess moderate physical activity using accelerometery in community dwelling women of color: A crosssectional study. International Journal of Behavioral Nutrition and Physical Activity. 2011:8(1):21. PubMed PMID: 21439052; PubMed Central PMCID: PMC3072301.

33. Lee RE, Mama SK, Medina AV, Reese-Smith JY, Banda JA, Layne CS, Baxter M, O'Connor DP, McNeill L, Estabrooks PA. Multiple measures of physical activity, dietary habits and weight status in African American and Hispanic or Latina Women. Journal of Community Health. 2011;36(6):1011-23. PubMed PMID: 21519867; PubMed Central PMCID: PMC4535354.

34. Lee RE, Medina AV, Mama SK, Reese-Smith JY, O'Connor DP, Brosnan M, Cubbin C, McMillan T, Estabrooks PA. Health is Power: An ecological theory-based health intervention for women of color. Contemporary Clinical Trials, 2011:32(6):916-23. PubMed PMID: 21782975; PubMed Central PMCID: PMC3185198.

35. Mama SK, Quill BE, Fernandez-Esquer ME, Reese-Smith JY, Banda JA, Lee RE. Body image and physical activity among African American and Hispanic or Latina women. Ethnicity \& Disease. 2011;21(3):281-287. PubMed PMID: 21942159.

36. McAlexander KM, Mama SK, Medina A, O'Connor DP, Lee RE. The concordance of directly and indirectly measured built environment attributes and physical activity adoption. International Journal of Behavioral Nutrition and Physical Activity. 2011;8(1):72. PubMed PMID: 21736740; PubMed Central PMCID: PMC3146893.

37. Lee RE, O'Connor DP, Smith-Ray R, Mama SK, Medina AV, ReeseSmith JY, Banda JA, Layne CS, Brosnan M, Cubbin C, McMillan T, Estabrooks PA. Mediating effects of group cohesion on physical activity and diet in women of color: Health Is Power. American Journal of Health Promotion. 2012;26(4):e116-e125. PubMEd PMID: 22375580; PubMed Central PMCID: PMC4535932.

38. Thomas S, Reading J, Shephard RJ. Revision of the physical activity readiness questionnaire (PAR-Q). Can J Sport Sci. 1992; 17(4): 338345.

39. Goldberg $\mathrm{JH}$, Kiernan M. Innovative techniques to address retention in a behavioral weight-loss trial. Health Educ Res. 2005; 20(4): 439447.

40. Maternal and Infant Health Assessment (MIHA) Survey 2015. Available at http://www.cdph.ca.gov/data/surveys/Pages/ MaternalandlnfantHealthAssessment\%28MIHA\%29survey.aspx.

41. Sarnoff R, Hughes D. Increasing health insurance coverage in the first year of life. Matern Child Health J. 2005; 9(4): 343-350.

42. American Fact Finder. Available at http://factfinder2.census.gov/ faces/nav/isf/pages/index.xhtml.

43. Pickering TG, Hall JE, Appel LJ, et al. Recommendations for blood pressure measurement in humans and experimental animals: part 1. blood pressure measurement in humans: a statement for professionals from the Subcommittee of Professional and Public Education of the American Heart Association Council on High Blood Pressure Research. Circulation. 2005; 111(5): 697-716.

44. Body Composition Analyzer/Scales 2014. Available at http:// www.tanita.com/BodyComposition.shtml.

45. SPSS 20.0 for Windows. Available at http://www.spss.com.

46. Mathew NT. Prophylaxis of migraine and mixed headache. A randomized controlled study. Headache. 1981; 21(3): 105-109.

47. Turk DC, Rudy TE. Neglected factors in chronic pain treatment outcome studies-referral patterns, failure to enter treatment, and attrition. Pain. 1990; 43(1): 7-25.

48. Greaney ML, Lees FD, Nigg CR, Saunders SD, Clark PG. Recruiting and retaining older adults for health promotion research: the experience of the SENIOR Project. J Nutr Elder. 2006; 25(3-4): 3-22.

49. Ismail MM, Gerrish K, Naisby A, Salway S, Chowbey P. Engaging minorities in researching sensitive health topics by using a participatory approach. Nurs Res. 2014; 22(2): 44-48.

50. Rogers IL, Johnson TR, Brown MB, Lantz PM, Greene A, Smith YR. Recruitment of women research participants: the Women's Health Registry at the University of Michigan. J Womens Health (Larchmt). 2007; 16(5): 721-728. 
51. Schmidt JA, Gruman C, King MB, Wolfson LI. Attrition in an exercise intervention: a comparison of early and later dropouts. J Am Geriatr Soc. 2000; 48(8): 952-960.

52. Warren-Findlow J, Prohaska TR, Freedman D. Challenges and opportunities in recruiting and retaining underrepresented populations into health promotion research. Gerontologist. 2003; 43(Spec No 1): 37-46.

53. Chen AH, Sallis JF, Castro CM, et al. A home-based behavioral intervention to promote walking in sedentary ethnic minority women: project WALK. Womens Health. 1998; 4(1): 19-39.

54. Lee RE, Soltero EG, Mama SK, Saavedra F, Ledoux TA, McNeill L. Developing a transcultural academic-community partnership to arrest obesity. Int Q Community Health Educ. 2014; 34(3): 215-233.

55. Barnett J, Aguilar S, Brittner M, Bonuck K. Recruiting and retaining low-income, multi-ethnic women into randomized controlled trials: successful strategies and staffing. Contemp Clin Trials. 2012; 33(5): 925-932.

56. Coulon SM, Wilson DK, Griffin S, et al. Formative process evaluation for implementing a social marketing intervention to increase walking among African Americans in the Positive Action for Today's Health trial. Am J Public Health. 2012; 102(12): 2315-2321.

57. King AC, Blair SN, Bild DE, et al. Determinants of physical activity and interventions in adults. Med Sci Sports Exerc. 1992; $24(6$ Suppl): S221-S236.

58. Li F, Harmer P, Glasgow R, et al. Translation of an effective tai chi intervention into a community-based falls-prevention program. $\mathrm{Am} J$ Public Health. 2008; 98(7): 1195-1198. 